\title{
Incongruent odors suppress perceptual categorization of visual objects: Behavioral and ERP evidence
}

Thomas Hörberg ${ }^{1,2}$, Maria Larsson ${ }^{1}$, Ingrid Ekström ${ }^{2,3}$, Camilla Sandöy ${ }^{1}$, \& Jonas K. Olofsson ${ }^{1}$

${ }^{1}$ Gösta Ekman Laboratory, Department of Psychology, Stockholm University

${ }^{2}$ Department of Linguistics, Stockholm University

${ }^{3}$ Aging Research Center (ARC), Karolinska Institute, Stockholm University 


\begin{abstract}
Visual stimuli often dominate non-visual stimuli during multisensory perception, and evidence suggests higher cognitive processes prioritize visual over non-visual stimuli during divided attention. Visual stimuli may therefore have privileged access to higher mental processing resources, relative to other senses, and should be disproportionally distracting when processing incongruent cross-sensory stimuli. We tested this assumption by comparing visual processing with olfaction, a "primitive" sensory channel that detects potentially hazardous chemicals by alerting attention. Behavioral and event-related brain potentials (ERPs) were assessed in a bimodal object categorization task with congruent or incongruent odor-picture pairings and a delayed auditory response target. For congruent pairings, accuracy was higher for visual compared to olfactory decisions. However, for incongruent pairings, reaction times (RTs) were faster for olfactory decisions, suggesting incongruent odors interfered more with visual decisions, thereby showing an "olfactory dominance effect". Categorization of incongruent pairings engendered a late "slow wave" ERP effect. Importantly, this effect had a later amplitude peak and longer latency during visual decisions, likely reflecting additional categorization effort for visual stimuli. In sum, contrary to what might be inferred from theories of "visual dominance", incongruent odors may in fact uniquely attract mental processing resources during perceptual incongruence.
\end{abstract}

\title{
Keywords
}

Visual dominance, olfaction, olfactory-visual processing, object categorization, event-related brain potentials 


\section{Introduction}

A long-standing notion in western thought is that visual impressions have an especially important role in human perception and cognition. For example, Aristotle wrote that vision "in its direct effects, is the superior sense" (Aristotle, trans. 2010). In a similar vein, Immanuel Kant postulated vision to be the noblest of all the senses, because it "has the widest sphere of perception in space" (Kuehn 2006:48). A strong emphasis on visual processing is also present in the neurocognitive literature. For more than 40 years, experiments have consistently shown that processing of visual stimuli "dominates" other senses in multisensory perception (e.g., Colavita 1974; Koppen and Spence 2007a; Sinnett, Spence and Soto-Faraco 2007; but see Ngo, Cadieux, Sinnett, Soto-Faraco and Spence 2011 for an exception). In a pioneering study, Colavita (1974) found that auditory stimuli often are neglected when presented concurrently with visual stimuli, an effect that has been replicated in more recent studies (e.g. Koppen and Spence 2007a; Sinnett et al. 2007). Similarly, in the "McGurk effect", the perception of speech sounds is influenced by incongruent visual-articulatory information (McGurk and MacDonald 1976; Traunmüller and Öhrström 2007). In visual-tactile multisensory decisions, participants rely more on visual than tactile information (e.g. Welch and Warren 1980) as visual stimuli distract from concurrent tactile stimulation (Hartcher-O'Brien, Levitan and Spence 2010). Visual input thus appears to be favored in multisensory perception. Higher neurocognitive processes also appear to prioritize visual over non-visual stimuli. When attention is divided between the visual and the auditory channel, processes related to response selection, as indexed by cortical responses, are strongly impaired for auditory but not for visual decisions (Hohnsbein, Falkenstein, Hoormann and Blanke 1991; Falkenstein, Hohnsbein, Hoormann and Blanke 1991).

The "visual dominance" effects have been interpreted in terms of selective attention; cognitiveperceptual resources are by default directed towards visual input compared to other sensory modalities (e.g., Posner, Nissen and Klein 1976; Sinnett et al. 2007). Other studies suggest visual dominance effects may instead be the result of the visual system actively inhibiting non-visual processes (Spence, Parise and Chen 2012).

Olfaction provides an interesting test of the visual dominance hypothesis. Although less wellinvestigated than "higher" senses, olfaction might serve as a powerful alerting cue to attract visual attention in order to achieve stimulus localization and identification (Herrick 1933). This alerting capacity of olfaction has been theorized as its defining feature (Köster, Møller and Mojet 2014). Incongruent odor cues might therefore provide disproportional influence over vision (i.e. dominance). While the awareness of an ambient odor can be effectively eliminated by engaging in a demanding visual perception task (Forster and Spence 2018), no previous study has directly compared how olfaction and vision compete for processing resources under conditions of equal task-relevance. Interactions between olfactory and visual perception are well-established; congruent visual information facilitates olfactory-based detection (Gottfried and Dolan 2003; Olofsson, Bowman and Gottfried 2013), discrimination (Dematte, Sanabria and Spence 2008), and identification (Höchenberger, Busch and Ohla 2015; Olofsson et al. 2013), and congruent olfactory information facilitates visual-based identification (Seigneuric, Durand, Jiang, Baudouin and Schaal 2010; Zhou, Jiang, He and Chen 2010) and motion perception (Kuang and Zhang 2015). In this preregistered study (Hörberg \& Olofsson 2018, osf.io/7qnwu/), we tested the generality of visual dominance by assessing task interference across olfactory-visual sensory channels.

We posited that perceptual dominance would involve "asymmetric" inhibition across sensory systems (Spence et al. 2012), mediated by the allocation of processing resources (e.g., Koppen and Spence 2007a; Posner et al. 1976; Sinnett et al. 2007). We devised a bimodal odor-picture categorization task with a delayed auditory target. Participants categorized familiar objects (lemon, pear, lavender, lilac) as fruits or flowers. The task was designed to be rapidly and accurately performed based on either olfactory or visual cues, following previous work (Olofsson, Bowman, Khatibi and Gottfried 2012; Olofsson, Hurley, Bowman, Bao, Mesulam and Gottfried 2014). On each trial, an odor and a picture were simultaneously presented. After a short delay, these cues were followed by a sound that indicated 
whether the fruit/flower decision should be based on the odor or the picture. In order to engage competition for processing resources, the odor-picture pair was incongruent on half of the trials (e.g. lemon odor, lavender picture). We assumed that when visual and olfactory stimuli activate two conflicting representations, the dominant sensory system will be less vulnerable to such cross-modal distraction and retain a relatively high behavioral performance. Incongruent stimuli in the dominant sensory modality should interfere more with behavioral decisions in the non-dominant modality than vice versa, resulting in a relative behavioral advantage during the incongruent condition for decisions in the dominant modality (see Figure 1).

As specified in our preregistration, we hypothesized that visual-based decisions should be overall faster and/or more accurate than olfactory-based decisions, even though the decision was triggered by a tone following a brief delay, which provided ample time for sensory processing of both odors and pictures (Olofsson et al. 2014; Olofsson, Syrjänen, Ekström, Larsson and Wiens 2018). In Figure 1, this general response advantage for visual stimuli over olfactory stimuli would be observed as a main effect of modality (upper panel). We further assumed, based on the vast literature on visual dominance, that if visual processing has a privileged access to attentional and working memory processing resources, incongruent visual stimuli should interfere more with olfactory categorization than the incongruent olfactory stimuli would interfere with visual categorization (middle panel). If, on the other hand olfactory processing dominates visual processing, incongruent olfactory stimuli should interfere more with visual categorization than vice versa (lower panel). In other words, a perceptual dominance account would predict an interaction effect between congruence and modality, and this would be observed in either reaction times (which are illustrated in Figure 1, right panels) or in response accuracies. Congruent odor-visual stimulus cues served as our control condition.

We also investigated cortical responses time-locked to the presentation of the auditory target, using event-related brain potentials (ERPs). This would allow us to draw further conclusions regarding the cortical processing sequence underlying perceptual dominance. We were interested in ERP effects that would correspond to behavioral effects and thereby aid interpretation of its cortical correlates. Further, we explored the notion that perceptual dominance involves asymmetric inhibition between perceptual

CONGRUENT

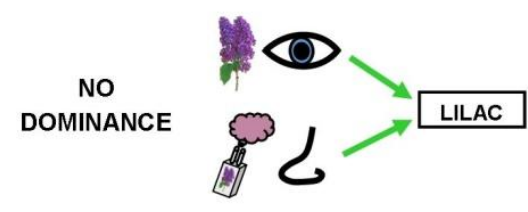

VISUAL DOMINANCE

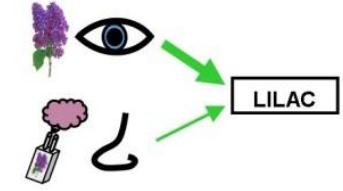

OLFACTORY DOMINANCE

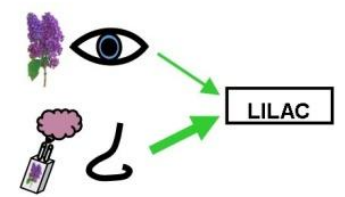

INCONGRUENT
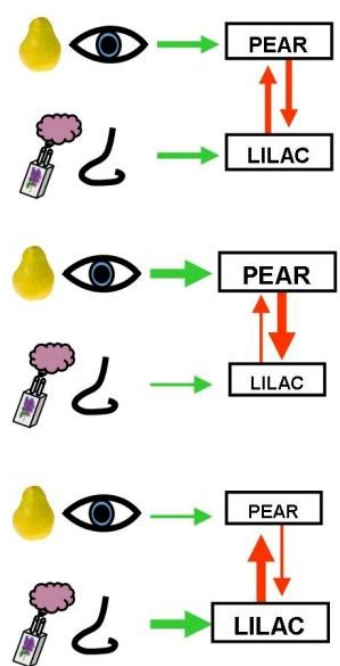

PREDICTED RESULTS
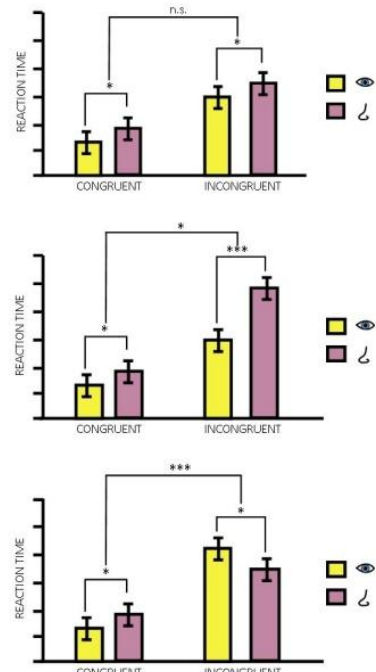

Figure 1. Illustration of how sensory dominance is conceptualized. Congruent visual-olfactory stimuli (e.g. lilac picture and lilac odor) leads to a bimodal activation of the representation of lilac, both under no dominance, visual dominance, or olfactory dominance. Incongruent visual-olfactory stimuli (e.g. pear picture and lilac odor), on the other hand, leads to competitive activation within each sensory modality, resulting in between-modality inhibition of the pear and lilac representations. Under no dominance, inhibition strength is symmetric, resulting in an equal interference effect on behavioral results. Under visual dominance, the olfactory representation is to a greater extent inhibited by the visual representation, resulting in delayed olfactory decisions. Under olfactory dominance, on the other hand, the olfactory representation has a stronger inhibitory effect on the visual representation, resulting in delayed visual decisions. 
systems. In our preregistration, we hypothesized that if perceptual dominance is a consequence of asymmetric inhibition, successful categorization of stimuli in the dominated sensory channel should require additional attentional resources needed to compensate for the strong interference effect of the incongruent input in the dominating channel ${ }^{1}$. The allocation of attentional resources during stimulus categorization has been linked to the P300 ERP response, a positive, centro-parietal or centro-frontal wave, that often peaks around $300 \mathrm{~ms}$ post stimulus presentation (e.g. Donchin 1981; Kutas, McCarthy and Donchin 1977; Picton 1992; Sutton, Braren, Zubin and John 1965; Verleger 1997; and Polich 20072011 for reviews). Stimulus categorization involves the integration of external stimuli with working memory representations, a process that is mediated by attention (Kok 2001). It has been suggested that P300 amplitude reflects the attentional demands of the categorization task (e.g. Kok 2001), which, in turn, co-occurs with inhibition of task-unrelated activity (Polich 2007). In the context of the present study, categorizing stimuli during cross-modal interference can therefore be expected to result in an enhanced P300 wave. Further, more attentional resources might be required to inhibit the sensory representation in the dominant sensory system. This should result in a more pronounced P300 wave during categorization in the non-dominant sensory channel.

\section{Materials and Methods}

\section{Participants}

Participants were recruited at Stockholm University via on-line advertisements. Participation was compensated with either gift vouchers worth 200 SEK or course credit. Power analyses based on behavioral and ERP pilot data and simulations (see supplementary materials) showed that a sample size of 40 individuals would be sufficient (power $>0.8$ ) even for small effect sizes (standardized $\beta \mathrm{s}=$ 0.2 ). The initial sample therefore consisted of 46 healthy adults who reported to have normal to corrected-to-normal vision and a normal sense of smell, who provided written informed consent and were screened for their ability to correctly identify the 4 stimulus odors. We excluded data from 10 participants who had less than $75 \%$ correct trials in either the congruent or incongruent conditions, and data from one participant with missing background data. The effective sample size therefore consisted of 35 individuals (M age: 31.3 years, range: 19-59 years, 16 females). In the ERP data analyses, data from an additional five participants was also excluded due to EEG artifacts. We chose to keep these five participants for behavioral analyses in order to preserve statistical power.

\section{Stimuli}

The stimuli consisted of four visual and four olfactory objects belonging to the categories fruit (lemon and pear) and flower (lavender and lilac). We chose odors that are easy to name and therefore primarily engage prefrontal language areas (i.e. cortical areas adjacent to inferior frontal gyrus) during working memory retention (Zelano et al. 2009). Odors were presented with an olfactometer that was controlled by the stimulus computer. We used $1-3$ milliliters of odor essences and oils from Stockholms Aeter \& Essencefabrik AB and Aroma Creative. The odor identification and rating tasks indicated that the odors were easy to identify and perceived as similar in intensity, pleasantness and specificity, but differed in edibility (see supplementary materials). The pictures were presented on a computer screen and consisted of photographed images that were matched in size, brightness, and hue. The auditory targets consisted of two $0.5 \mathrm{~s}$ long sinus tone that were presented in earphones. Tone amplitudes were adjusted for tone loudness. The low tone consisted of a $630 \mathrm{~Hz}$ tone with $60.8 \mathrm{~dB}$ and the high tone of $1250 \mathrm{~Hz}$ tone with $62.2 \mathrm{~dB}$.

\section{Procedure}

The experiment was conducted in brightly lit and well-ventilated olfactory testing room at the Gösta Ekman laboratory, Department of Psychology, at Stockholm University. Participants were informed

\footnotetext{
${ }^{1}$ See supplementary materials for an extended discussion regarding the neurophysiological processes underlying the experimental task and the preregistered, predicted ERP effects.
} 
about the experiment and that they could abort it at any time. They were seated at a one-meter distance from the stimulus computer screen. Participants performed a standardized protocol to ensure that the experimental stimuli were correctly identified. Also, participants rated the perceived perceptual features (see supplemental text for details) of the odors.

In the main experimental task, participants categorized visual or olfactory stimuli as fruit or flower. In order to investigate modality dominance in terms of interference asymmetry, we used a categorization task with cross-modal interference. Visual and olfactory stimuli were presented simultaneously. On congruent trials, the same visual and olfactory objects were used (e.g., picture and odor of pear). On incongruent trials, objects from each of the two categories were used (e.g., picture of pear and odor of lilac). Importantly, in order to remove bias due to processing speed differences between visual and olfactory perception, the auditory target cues that informed about the object target (i.e. picture or odor), were delayed by a varying interval of 1000-2000 ms post stimuli offset. This allowed for statistical analyses of the possible effect of lag time. Further, the timing of the onset of the cues, and the delayed auditory target, ensures that sensory processing speed should not influence the results (see supplementary materials).

A categorization trial is illustrated in Figure 2. Each trial begun with the presentation of the odor. First, a black fixation cross appeared for $1500 \mathrm{~ms}$ in the center of the screen. It indicated that an odor was about to be presented and that it was time to prepare for the sniff by inhaling. Following a $200 \mathrm{~ms}$ blank screen, a red fixation cross appeared that indicated that it was time to sniff. The odor was simultaneously presented by the olfactometer. At $400 \mathrm{~ms}$ after olfactory stimulation onset, the picture appeared in the center of the screen. It was presented together with the odor for $1500 \mathrm{~ms}$. In other words, pictures were presented with a lag of $400 \mathrm{~ms}$ relative to the odors in order to compensate for the additional processing time of olfactory stimuli, in comparison to that of visual stimuli (asevidenced by, for instance, the $400 \mathrm{~ms}$ lag in P300 latency between visual and olfactory oddball tasks, see e.g., Alexander et al. 1995; Pause, Sojka, Krauel and Ferstl 1996, and supplementary materials for further discussion). After stimulus presentation, the screen turned blank for 1000-2000 ms. Following this

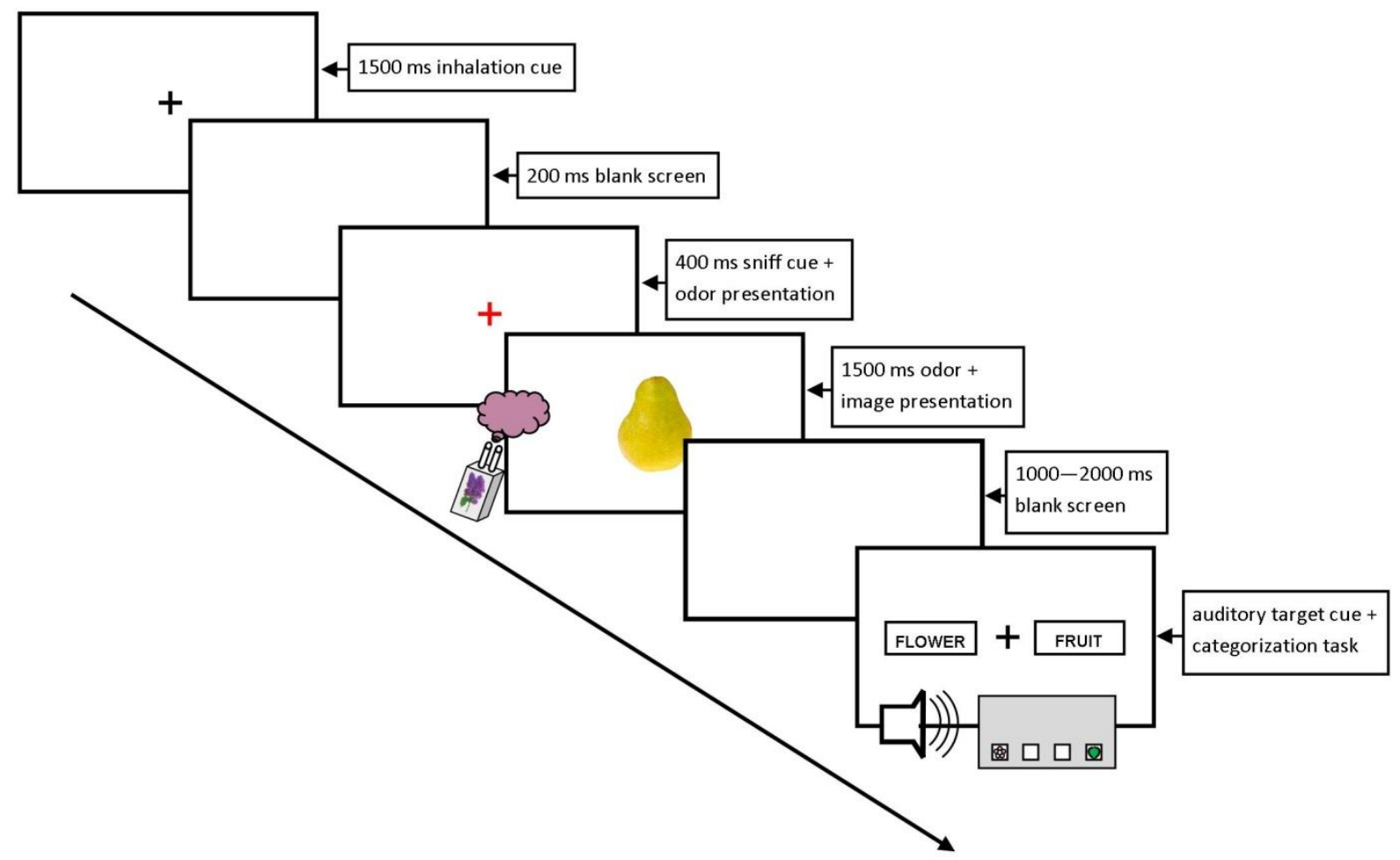

Figure 2. Trial structure of the categorization task. During training trials (but not in experimental trials), the object modality to be categorized was also displayed in the final screen, written above the fixation cross, in order to learn the meaning of the two target tones. 
delay, participants performed the categorization task. The target cues, i.e. either the low or the high sinus tone, were presented simultaneously as a black fixation cross appeared on the center of the screen. The fixation cross was flanked by two text boxes that reminded about the button assignment (i.e., whether the left button was used for fruits and the right for flowers, or vice versa). The position of the boxes (left vs. right) corresponded to the button assignment. The task was performed by pressing either the leftmost or the rightmost button of a four-button response box. Participants were encouraged to respond as quickly and accurately as possible. The four possible combinations of tone and button assignment was counterbalanced across participants. Each trial ended with a delay (minimum $1000 \mathrm{~ms}$ ) that ensured that at least 10 seconds had past since the start of the trial.

Participants conducted 128 trials, 2 (congruence) $\times 2$ (modality) $\times 2$ (category) $\times 2$ (stimuli) $\times 8$ (repetitions), evenly distributed across 4 blocks. In the incongruent trials, the target stimulus object (i.e., the sensory object to be categorized) co-occurred with either of the two incongruent stimuli equally often (e.g. pear odor co-occurred with lilac and lavender pictures on an equal number of trials). Trial presentation order within a block was randomized. At the beginning of each block, a visual display informed that the next block was about to start. The block started with a button press. In order to get familiarized with task, participants performed a training session consisting of 16 trials, 2 (congruence) $\times 2$ (modality) $\times 2$ (category) $\times 2$ (stimuli), before the actual experiment started. In the training trials, but not the experimental trials, the sensory modality to categorize was displayed on the screen (directly above the fixation cross, see Figure 2), in order for the participants to learn the meaning of the target tones. Participants were encouraged to take short breaks in between blocks. They were told to avoid blinking and moving from the time of the inhalation cue was presented to the time of their response. The study was approved by a regional ethics board in Stockholm (2014/2129-31/2).

\section{Apparatus}

Odors were presented with a custom-built, continuous-flow olfactometer described in detail in Lundström et al. (2010). The olfactometer can easily be controlled from experimental software such as Psychopy through the parallel port and provides a fast and invariant temporal resolution (approximately $160 \pm 2 \mathrm{~ms}$ from the software trigger timing to odor delivery), emits a stable odor concentration over time (approximately a $0.5 \%$ decrease over a 10-minute period), and has been shown to be suitable for recording olfactory ERPs (Lundström et al. 2010).

Visual stimuli were presented on a 24" Benq XL2430-B TN-screen with $100 \mathrm{~Hz}$ refresh rate and a resolution of $1920 \times 1080$ pixels. The experiment was run on a Windows 7 PC. In the odor identification and rating tasks, participants responded with the mouse. In the categorization task, they responded with a Cedrus RB-740 Response Box (Cedrus Corporation, San Pedro, CA, USA).

\section{EEG recording}

EEG was recorded with a 64 pin electrode Active Two Biosemi system (Biosemi, Amsterdam, Netherlands), using EEG caps (Electro-Cap International, Eaton, OH). In addition to the 64 10-20 electrodes, the Biosemi system uses an internal reference electrode (CMS), positioned in between PO3 and POz, and a ground electrode (DRL) positioned in between POz and PO4. EOG was recorded with two flat electrodes attached with an adhesive disk, one positioned at the outer canthus of the right eye, and the other directly below the right eye. Data was sampled at $2048 \mathrm{~Hz}$ with a hardware lowpass filter at $410 \mathrm{~Hz}$, but downsampled to $512 \mathrm{~Hz}$ offline.

\section{Data analysis}

\section{EEG preprocessing}

We performed all offline EEG preprocessing with the EEGLAB toolbox (Delorme and Makeig 2004) in MATLAB (The MathWorks, Inc.). First, the continuous data was epoched into segments lasting from $1000 \mathrm{~ms}$ before each trial to $1000 \mathrm{~ms}$ after each response, with training session trials included. The data was downsampled to $512 \mathrm{~Hz}$ and filtered with a 0.2 highpass filter and $40 \mathrm{~Hz}$ lowpass filter. 
Bad channels were identified using a custom-made script and the clean_channels() function in the clean_rawdata EEGLAB plugin. Channels were considered bad if the amplitude difference exceeded $500 \mathrm{mV}$ in more than $50 \%$ of $1000 \mathrm{~ms}$ time windows, if their correlation with their robust estimates as calculated from the signal of the 16 neighboring channels was less than 0.75 , or if their signal-to-noise ratio deviated with more than 4 standard deviations from the channel mean signal-to-noise ratio of all channels. Bad channels were then interpolated using spherical splines, and the data was re-referenced to the average of all channels, using a full rank average ${ }^{2}$. We then performed ocular artifact rejection on the basis of Independent Components Analysis (ICA). The data used for ICA decomposition was highpass filtered at $1 \mathrm{~Hz}$, trimmed of noisy data by visual inspection, and then analyzed with the AMICA eeglab plugin. The resulting ICA components were then transferred back to the original 0.2 $\mathrm{Hz}$ highpass-filtered data. Ocular artifact ICA components were automatically identified and removed using the icablinkmetrics plugin (Pontifex, Miskovic and Laszlo 2017). The plugin identifies artifactual components on the basis of 1) the similarity between the mean eyeblink activity in an EOG channel or a EOG channel group, on the one hand, and the component mean activity, on the other, in terms of their correlation, 2) the overlap in terms of morphology and amplitude between the mean eyeblink activity in the EOG channel(s) and the component mean activity in terms of their normalized convolution, and 3) the reduction in the mean eyeblink artifact when the component is removed. Components were identified on the basis of their relationships with the activity in either the vertical EOG channel, the horizontal EOG channel, or the mean of channels Fp1, AF7, FCz, Fp2, and AF8. We used a correlation coefficient threshold of 0.9 , a convolution coefficient threshold of 0.1 , and an artifact reduction threshold of $10 \%$. In order for a component to be classified as artifactual, all three metrics also had to be statistically significant at the .001 alpha level. On average, 3 components were identified as artifactual in each participant data set (min: 0 , max: 6).

The artifact-corrected data from the critical trials was divided into single trial epochs of $-200 \mathrm{~ms}$ to $1000 \mathrm{~ms}$ relative to the onset of the visual stimuli, and the onset of auditory targets, with $200 \mathrm{~ms}$ used for baseline correction. Epochs time-locked to the presentation of the visual stimuli were differentiated on the basis Congruence (i.e., Congruent vs. Incongruent), and epochs time-locked to the auditory targets on the basis of Congruence and Modality (i.e., the four conditions henceforth referred to as Olfactory-congruent, Visual-congruent, Olfactory-incongruent and Visual-incongruent). Epochs were defined as noisy and removed if they had a $\pm 120 \mathrm{uV}$ amplitude difference in any EEG channel, the amplitude difference in any of the EEG channels deviated by more than 6 SDs from the mean amplitude channel difference in all epochs, or if the amplitude difference of four channels deviated by more than 4 SDs from their mean channel amplitude differences in all epochs. We also excluded epochs from trials with RTs below $200 \mathrm{~ms}$ or above $5000 \mathrm{~ms}$ (similar to Olofsson et al. 2012). Subjects with less than 15 epochs remaining in any condition were excluded from subsequent data analyses, resulting in the exclusion of 5 participants.

\section{Statistical analyses}

All results were analyzed in the statistical software R (R Core Development Team 2018), using the ERP data analysis toolkit Fishermans Friend (Hörberg, forthcoming). As stated in the preregistration, we performed Bayesian mixed effects modeling in the Stan modeling language (Stan Development Team 2017), using the R package Rstan (Stan Development Team 2018). RTs and ERP amplitudes were analyzed with linear mixed effects modeling, and accuracy with logistic mixed effects modeling. Full model specifications and model priors are presented in the supplementary materials. Inferences about parameter effects (e.g., the Congruence $\times$ Modality interaction) were done on the basis of the parameter Credability Intervals (CIs). We consider a parameter 95\% CI not including zero as evidence for an effect of the parameter at hand. We also report the posterior probability $(p)$ of a parameter being zero or taking on values in the opposite direction of the mean parameter estimate, divided by 2 . All Bayesian analyses were complemented with frequentist mixed effects modeling, reported in the

\footnotetext{
${ }^{2}$ Before averaging, we included a dummy channel with zero activity that was removed after averaging. This is done in order to avoid rank deficiency, which can affect the subsequent ICA decomposition.
} 
supplementary materials. All models contained fixed effects for the independent variables Congruence (Congruent vs. Incongruent), Modality (Visual vs. Olfactory) and for the Congruence $\times$ Modality interaction. The models also included fixed effects for the following potential confounders.

Trial number. In order to control for any learning effects remaining after the initial training session, we included trial number as a control variable.

Delay. The varying delay in ms between object presentation and the presentation of the auditory target was also included. A longer delay give participants more time for stimulus processing and response preparation, and might thus result in shorter response times and higher accuracies.

Object category. The object category - fruit or flower - was also included in order to control for any potential differences in the categorization of fruits versus the categorization of flowers.

Gender. The gender of the participant was also included as some studies have found women to have somewhat superior olfactory perceptual abilities than men (e.g. see Doty \& Cameron 2009 for a review).

Similarity index. In order to control for a potential influence of between-modality differences in perceived stimulus similarity, we also included a between-category similarity index as a control variable. This index aimed to capture the participant-specific between-category similarity, that is, the individually perceived similarity between a cue category stimulus (e.g., lemon of the fruit category) and the two stimuli of the other, competing category (i.e., lilac and lavender of the flower category). We wanted to quantify whether a high between-category similarity could render the categorization task more difficult, as the cue stimulus should be harder to differentiate from the stimuli of the competitor category. Within-category similarity, on the other hand, should not influence categorization, since a confound (e.g., confounding pear and lemon) would still enable a categorical decision (fruit). This index was calculated on the basis of between-category similarity ratings of the stimuli (see supplementary materials). First, in order to make similarity ratings comparable across participants, ratings were standardized within participants, ensuring that participant rating means and standard deviations were the same for each participant. The between-category similarity index was then calculated within each participant, cue stimulus and modality as the mean of the standardized similarity ratings involving the stimulus at hand. Since participants rated the stimuli for their similarity to each of the two competitor category stimuli twice (e.g., two similarity ratings of lemon-lilac, and two of lemon-lavender), this index was the mean of four ratings.

All models also include random intercepts for participants and items, the latter differentiating between all possible visual and olfactory stimuli combinations. Thereby, we control for any systematic differences between subjects and stimuli pairs. We also included a by-participants random slope for Trial number, thereby controlling for any differences in learning between participants. RT data was log-transformed in order to ensure normality. All continuous covariates were standardized by subtracting the mean, and, following Gelman (2006), dividing by two standard deviations. Categorical variables were effect-coded through centering ${ }^{3}$. Main effects are therefore tested against the grand mean of the data. In the event of significant Congruence $\times$ Modality interactions, we conducted simple effect follow-up analyses, testing the effect of Modality in the congruent and the incongruent conditions separately. This was done by including three dummy coded predictors either for Visualcongruent, Olfactory-congruent and Visual-incongruent (testing Modality within incongruent trials), or for Visual-incongruent, Olfactory-incongruent and Visual-incongruent (testing Modality within congruent trials).

\footnotetext{
${ }^{3}$ That is, by coding the variable as 0 or 1 and subtracting the mean, see e.g. Gelman and Hill (2006: 55).
} 


\section{ERP analyses}

ERP data was analyzed with Bayesian and frequentist linear mixed effects models. As such, these analyses were conducted on single trial (rather than subject average) mean ERP amplitudes across time windows and electrode groups (similar to e.g. Frömer, Maier and Abdel Rahman 2018). We conducted analyses on ERPs time locked to stimulus presentation (reported in the supplementary materials), on the one hand, and to the auditory target, on the other. Since this is the first study of its kind to include ERP data, we did not make any specific predictions in terms of exact time windows and regions of interest (ROIs). These were chosen on the basis of previous literature and visual inspection of the data. We performed analyses on mean amplitudes in the P300 time window, ranging from 320-550 ms, across the centro-frontal (CF) scalp region which consisted of electrodes AF3, Afz, $\mathrm{AF} 4, \mathrm{~F} 1, \mathrm{Fz}, \mathrm{F} 2, \mathrm{FC} 1, \mathrm{FCz}, \mathrm{FC} 2$. We also conducted three separate analyses in the late time windows, 600-700, 700-800, and 800-900 ms, across the centro-occipital (CO) region, consisting of P4, P2, Pz, P1, P3, PO8, PO4, POz, PO3, PO7, O2, Oz, O1 (see Figure A8 in supplementary materials).

\section{Results}

In the following, we first report analyses of behavioral data and then analyses of ERP data. We only report significant results.

\section{Behavioral data}

Reaction times

Mean RTs from each condition (i.e., Olfactory-congruent, Visual-congruent, Olfactory-incongruent and Visual-incongruent) are shown in Figure 3. The results of the Bayesian linear mixed effects models of $\log$ RTs are shown in Table 1. As expected, categorization RTs are faster for congruent than for incongruent stimuli, as confirmed by the fact that the CI of Congruence does not include zero. Further, RT:s during visual-based responses are slower than olfactory-based responses when the stimuli are incongruent. Although there is main effect of Modality, and therefore a general RT

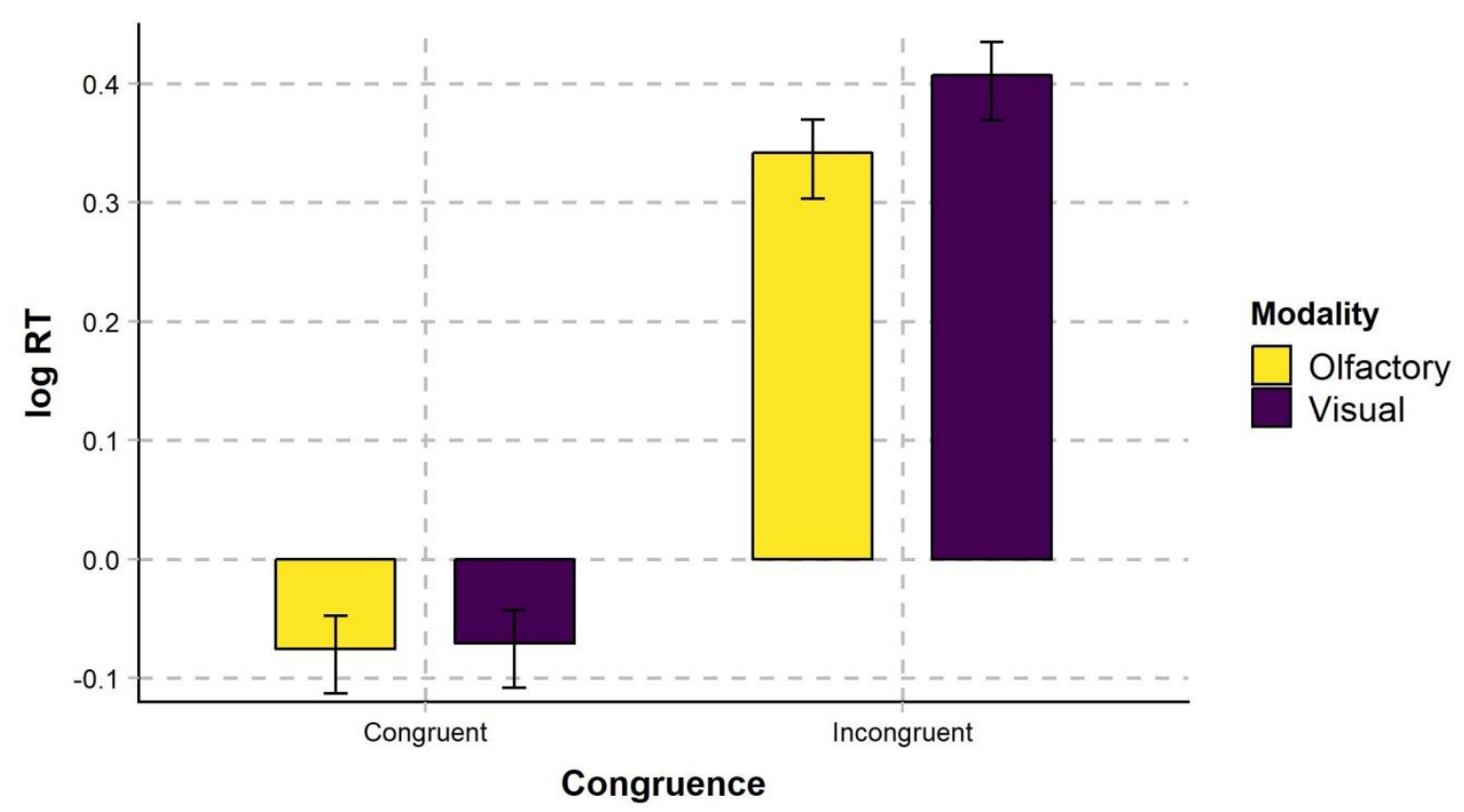

Figure 3. Log RTs (uncorrected for accuracy), differentiated on the basis of Congruence (Congruent vs. Incongruent) and the Modality of the target stimulus object (Olfactory vs. Visual). Error bars show 95\% CIs as estimated by the Bayesian model. 
Table 1. Results of the Bayesian linear mixed effects models of log RT data. P-values are the percentage of parameter samples being zero or taking on values in the opposite direction of the mean parameter estimate, out of a 4000 samples parameter posterior distribution.

\begin{tabular}{lrrrrr}
\hline Parameter & Beta & S. E. & Cl lower & Cl upper & $\boldsymbol{p}$ \\
\hline Intercept & 0.17 & 0.07 & 0.04 & 0.30 & .009 \\
\hline Congruence & 0.45 & 0.03 & 0.38 & 0.52 & $<.0001$ \\
Modality & 0.04 & 0.02 & 0.00 & 0.08 & .041 \\
Congruence $\times$ & 0.06 & 0.03 & 0.00 & 0.11 & .041 \\
Modality & 0.02 & 0.02 & -0.02 & 0.05 & .383 \\
\hline Category & 0.14 & 0.13 & -0.11 & 0.40 & .280 \\
Sex & -0.35 & 0.03 & -0.42 & -0.28 & $<.0001$ \\
Trial & 0.02 & 0.01 & -0.01 & 0.04 & .229 \\
Delay & 0.01 & 0.02 & -0.03 & 0.05 & .516 \\
\hline Similarity & & & & &
\end{tabular}

difference between modalities, there is also a Congruence $\times$ Modality interaction effect. Bayesian follow-up simple effects analyses did not find a modality difference within congruent trials, but instead within the incongruent trials, $\beta=0.07$, S.E. $=0.03, \mathrm{CI}=[0.02,0.12], \mathrm{p}=.009$. Overall then, RTs do not differ between olfactory and visual categorizations when the cues are congruent. When the cues are incongruent, on the other hand, RTs are on average $70 \mathrm{~ms}$ faster for olfactory than for visual categorizations, indicating a higher degree of olfactory interference with visual categorization, than vice versa.

\section{Accuracy}

Accuracy of each condition is shown in Figure 4. The results of the Bayesian logistic mixed effects model on the accuracy data is shown in Table 2. As expected, accuracy is higher for congruent than for incongruent stimuli, as shown by the Congruence main effect. Further, accuracy is higher for visual targets when the stimuli are congruent, but do not differ between modalities when the stimuli

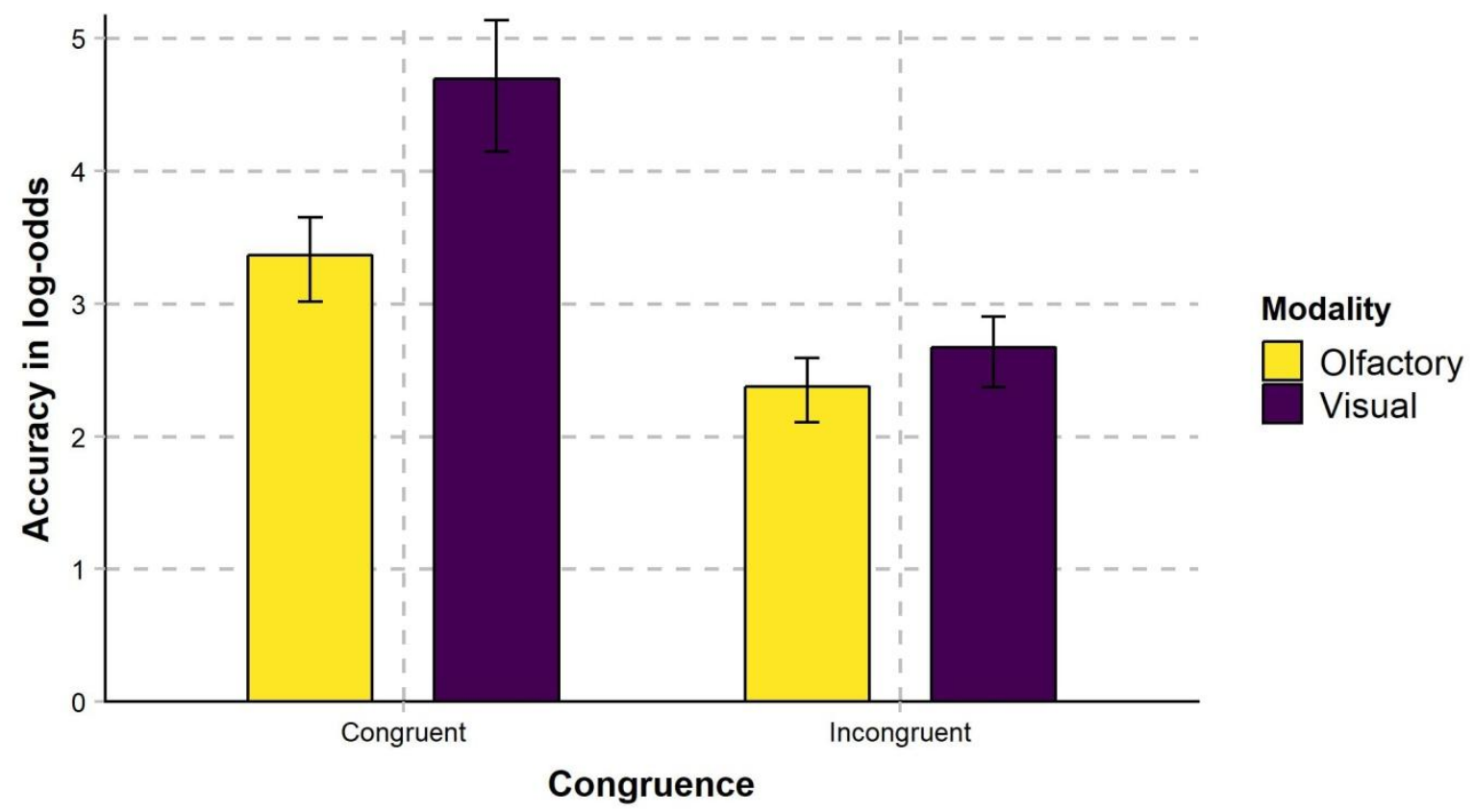

Figure 4. Accuracy in log-odds, differentiated on the basis of Congruence (Congruent vs. Incongruent) and the Modality of the target stimulus object (Olfactory vs. Visual). Error bars show 95\% CIs as estimated by the Bayesian model. 
Table 2. Results of the Bayesian logistic mixed effects models of accuracy data. P-values are the percentage of parameter samples being zero or taking on values in the opposite direction of the mean parameter estimate, out of a 4000 samples parameter posterior distribution.

\begin{tabular}{lrrrrr}
\hline Parameter & Beta & S. E. & Cl lower & Cl upper & $\boldsymbol{p}$ \\
\hline Intercept & 3.68 & 0.26 & 3.18 & 4.18 & $<.0001$ \\
\hline Congruence & -1.34 & 0.33 & -1.96 & -0.63 & .001 \\
Modality & 0.58 & 0.24 & 0.11 & 1.07 & .012 \\
Congruence $\times$ & -0.8 & 0.35 & -1.49 & -0.11 & .023 \\
Modality & 0.19 & 0.17 & -0.13 & 0.52 & .252 \\
Category & -0.31 & 0.37 & -1.07 & 0.46 & .391 \\
Sex & 0.54 & 0.24 & 0.07 & 1.04 & .020 \\
Trial & -0.12 & 0.15 & -0.4 & 0.18 & .426 \\
Delay & -0.26 & 0.21 & -0.68 & 0.17 & .210 \\
\hline Similarity &
\end{tabular}

are incongruent. There is a Modality main effect and therefore a general difference in accuracy between modalities. However, there is also a Congruence $\times$ Modality interaction effect. Follow-up simple effects analyses showed that accuracy is higher for visual targets in the congruent trials, $\beta=$ 1.13 , S.E. $=0.36, \mathrm{CI}=[0.44,1.83], p=.001$, but that there is no accuracy difference between modalities in the incongruent trials.

Taken together, although visual stimuli are categorized more accurately when the stimuli are congruent, there is no categorization advantage for visual stimuli in the incongruent conditions, neither in terms of RTs nor in terms of accuracy. That is, our behavioral data do not provide any evidence for overall visual dominance when incongruent odors are presented. Instead, incongruent odors seem to interfere with visual decisions more than vice versa, as indicated by the RT advantage for incongruent olfactory targets in comparison to incongruent visual targets. Contrary to the well-established notion that visual processing dominates other sensory input, these findings instead suggest that olfactory input can dominate visual input under some circumstances.

\section{ERP data}

Given our behavioral results, we investigated corresponding ERP effects time-locked to the auditory target that were related to the increased processing demands of incongruent compared to congruent trials, and their observed interactions with sensory modality. Stimulus presentation ERPs are further discussed in the supplementary materials. Grand average auditory target ERP:s across the CF and CO ROIs together with topoplots of congruent-incongruent grand average differences in the P300 (320$550 \mathrm{~ms}$; Figure 5, illustrating the fronto-central effect) and the positive slow wave (PSW; 600-700, 700-800 and 800-900 ms; Figure 6, illustrating the centro-occipital effect). In the following, we first present analyses regarding the P300, followed by the PSW effects.

\section{P300 (320-580 ms)}

The analysis in the P300 time window in the CF ROI showed a main effect of Congruence, $\beta=0.65$, S.E. $=0.16, \mathrm{CI}=[0.33,0.97], p=.001$, but no Congruence $\times$ Modality interaction. In other words, incongruent trials elicit a fronto-centrally distributed positivity in the P300 time window which is independent of modality of the target stimulus object.

PSW (600-900 ms)

As shown in Figure 6, incongruent trials engender a more pronounced positive slow wave in the late, 600-900 ms time window in the pareito-occipital region, an effect that is likely to reflect increased processing demands. Importantly, this effect differs between modalities in that it has a later amplitude peak and longer latency when the target stimulus object is visual than when it is olfactory (see Figure 


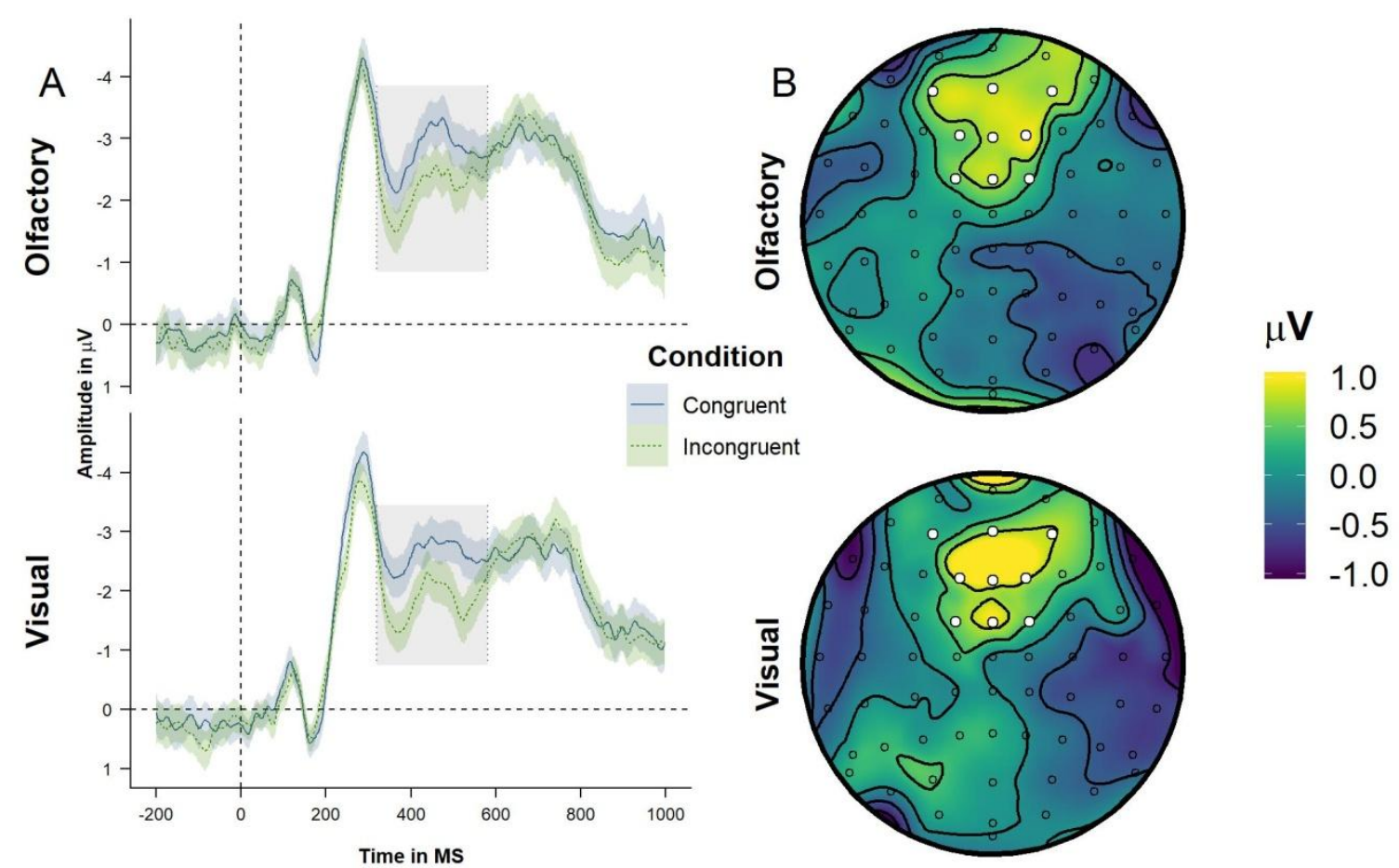

Figure 5. Panel A. Grand average ERPs time-locked to the presentation of the audio probes, averaged across the CF ROI, and differentiated on the basis of Congruence and Modality. Shaded areas show 95\% confidence intervals. Grey areas mark the 320-550 ms time window. Panel B. Topography of incongruent-congruent grand average difference of the P300 (320-550 ms) time window.

6). We conducted three separate analyses on ERP amplitude averages in each of the three consecutive $100 \mathrm{~ms}$ windows. In the 600-700 ms latency range, we found a main effect of Congruence, $\beta=0.70$, S.E. $=0.18, \mathrm{CI}=[0.34,1.06], p=.001$, but no Congruence $\times$ Modality interaction effect, similar to the $\mathrm{P} 300$ results. In the 700-800 ms time window, the Congruence main effect was also present, $\beta=0.59$, S.E. $=0.18, \mathrm{CI}=[0.23,0.96], p=.004$, but, importantly, it was complemented by a Congruence $\times$ Modality interaction, $\beta=0.66$, S.E. $=0.29, \mathrm{CI}=[0.10,1.24], p=.023$. In the $800-900 \mathrm{~ms}$ time window, finally, there was no main effect of Congruence, but instead a main effect of Modality, $\beta=$ 0.46 , S.E. $=0.23, \mathrm{CI}=[0.02,0.90], p=.043$, as well as a Congruence $\times$ Modality interaction, $\beta=$ 0.90 , S.E. $=0.29, \mathrm{CI}=[0.32,1.48], p=.003$. Follow-up simple effects analyses showed no Modality differences in the 600-700 ms time window. In the 700-800 and 800-900 ms time windows, on the other hand, there was a Modality difference in the Incongruent condition, [700-800 ms: $\beta=0.72$, S.E. $=0.26, \mathrm{CI}=[0.22,1.22], p=.003,800-900 \mathrm{~ms}: \beta=0.91, \mathrm{~S} . \mathrm{E} .=0.27, \mathrm{CI}=[0.39,1.44], p=.001]$, but not in the Congruent condition. These findings suggest additional costs during the categorization of visual stimuli when distracted by the presence of incongruent odors, compared to vice versa. This asymmetrical interference is present in terms of a late and temporally extended interference period from 700-900 ms after target onset. These findings therefore complement the behavioral results in that they indicate a substantial olfactory interference on visual categorizations.

\section{Discussion}

A well-established assumption in psychological research is that visual stimulus processing "dominates" processing in other sensory modalities. Visual dominance has been suggested to involve asymmetric inhibition between sensory systems (e.g. Spence et al. 2012). If visual processes generally inhibits non-visual processes, this should hold true also in categorization tasks with bimodal stimuli. We designed an odor-picture categorization task with cross-modal interference, testing the assumption of visual dominance using olfaction as a novel modality of comparison. Strikingly, our results provide converging behavioral and neurophysiological evidence of olfactory, rather than visual, dominance; incongruent odors exert more influence on visual processing, than vice versa. 

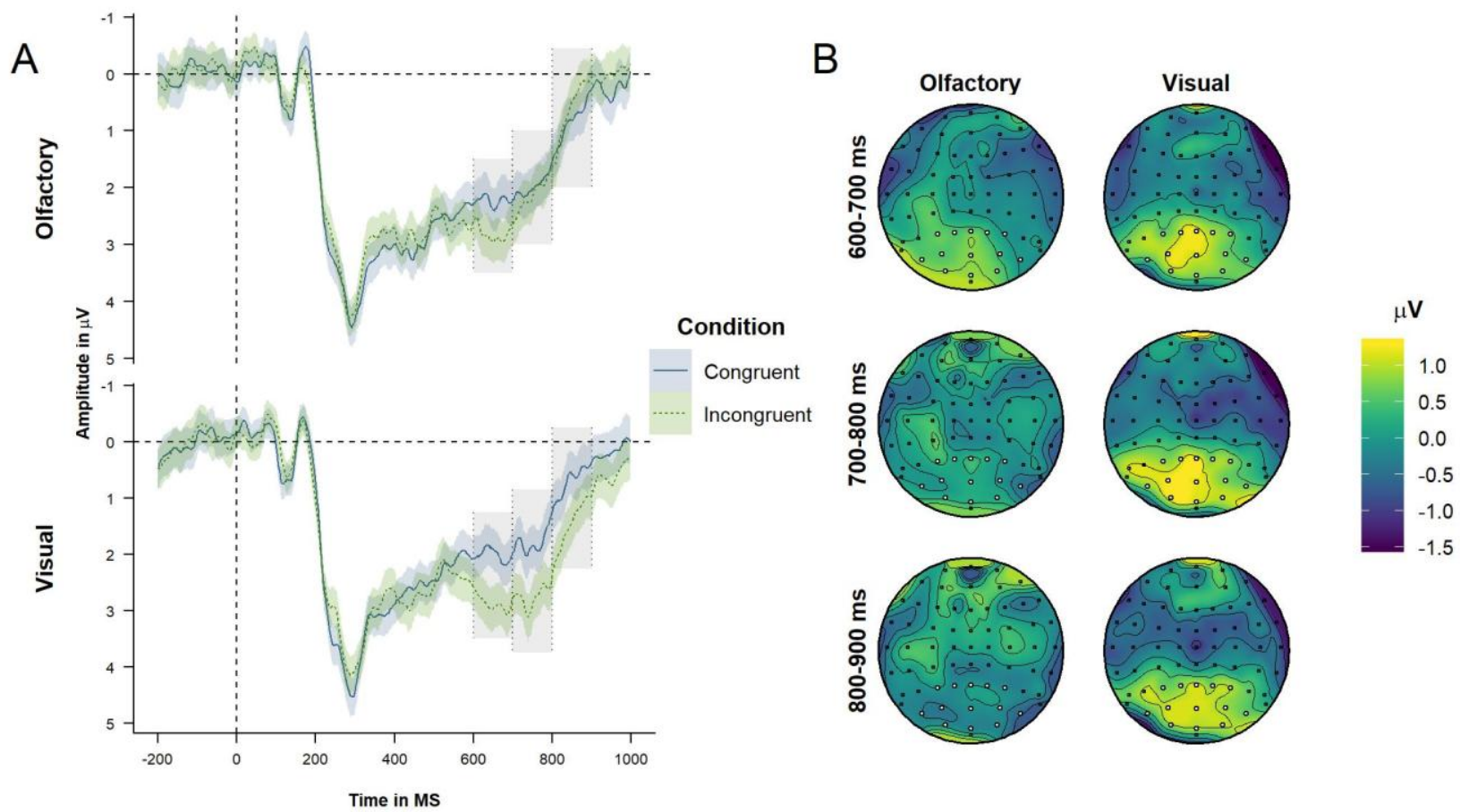

Figure 6. Panel A. Grand average ERPs time-locked to the presentation of the auditory targets, averaged across the CO ROI, and differentiated on the basis of Congruence and Modality. Shaded areas show 95\% confidence intervals. Grey areas mark the 600-700, 700-800, and 800-900 ms time windows. Panel B. Topography of incongruent-congruent grand average difference of the 600-700, 700-800 and 800-900 ms time windows.

Our behavioral findings may provide new insights regarding the complimentary roles of the senses. In particular, when visual and olfactory inputs are congruent, visual processing indeed offers a speed and accuracy advantage over olfaction, as would be expected by a purportedly dominant sensory system. But during incongruent input, it is instead olfactory cues that are surprisingly distracting, effectively impairing visual categorizations by disproportionally delaying responses and reducing their accuracy. The results of our study provide support for the notion that a foundational characteristic of olfaction is to effectively attract attention and enable processing in other senses. Anatomist C.J. Herrick (1933) postulated that mammalian brain evolution was characterized by a rapid growth in visual, but not olfactory cortical expansion, such that human olfaction would effectively provide alerting cues for further visual processing. More recently, Köster et al. (2014) suggested that the olfactory system is particularly sensitive to contextually inappropriate odors, which might serve as an especially alerting cue to other senses, but is rather indifferent to expected odors. In a similar vein, our findings show a behavioral advantage for olfactory decisions only when odor and picture cues are incongruent. In other words, it is only when odors are inappropriate relative to the visual information that odors receive privileged processing.

ERP effects provided valuable information about the cortical processing sequence underlying our behavioral observations. We were particularly interested in the idea that dominance stems from asymmetric inhibition between sensory systems (e.g., Spence et al. 2012). We reasoned that if asymmetric inhibition is responsible for sensory dominance, participants would require additional processing resources to compensate for the strong interference effect caused by incongruent input in the dominant modality. In line with this notion, we found a cortical processing sequence that started with overall effects of stimulus incongruency, gradually giving way to modality interactions that support our behavioral results of olfactory dominance. First, a more pronounced fronto-central P300 wave was observed during the categorization of incongruent stimuli. As the P300 amplitude is related to the mobilization of attentional resources during categorization tasks (e.g. Kok 2001), we interpret this P300 effect to reflect the additional attentional resources needed to inhibit the interfering percept during the incongruent condition. Our behavioral interaction effects were reflected in a late, centro- 
occipital positive slow wave in the incongruent condition. Importantly, this effect had a later amplitude peak and longer latency for visual categorizations (peak $738 \mathrm{~ms}$, latency 600-900 ms) than for olfactory categorizations (peak $673 \mathrm{~ms}$, latency 600-700 ms). In other words, these findings provide evidence for olfactory dominance over visual input also in terms of differences in cortical activity. Positive slow wave activity has been found in tasks where target identification prompts the execution of additional tasks (García-Larrea and Cézanne-Bert 1998). In the congruent condition, the categorization response can be prepared already before the auditory target. In the incongruent condition, on the other hand, the task consists of categorizing the auditory target, selecting between visual and olfactory object working memory representations, and finally making a categorization response based on the selected working memory representation. Positive slow wave activity has been linked to such response decision costs (Kok and De Jong 1980; Ruchkin and Sutton 1983; Ruchkin, Sutton, Kietzman and Silver 1980), response selection (Falkenstein, Hohnsbein and Hoormann 1993, 1994), working memory updating operations prompted by a secondary task (Johnson and Donchin 1985), sustained attention to task performance (Gevins et al. 1996), or working memory load during retrieval (García-Larrea and Cézanne-Bert 1998; Honda et al. 1996). Thus, although several accounts of late positive slow waves have been suggested, including the proposal that they reflect the completion of a broad array of task-dependent cognitive operations following target detection (GarcíaLarrea and Cézanne-Bert 1998), it seems clear that slow wave latency and amplitude reflect processing speed and processing effort. Our ERP findings therefore complement the behavioral results in that they show additional categorization costs for visual stimuli compared olfactory stimuli during crossmodal interference - thus indicating that incongruent olfactory stimuli interfere with visual categorization decisions more than vice versa. It should be pointed out that our study differs from earlier studies of sensory dominance in that it investigates sensory dominance in terms of inhibition between representations on the category level. Whereas earlier studies were concerned with mere stimulus detection (Koppen, Alsius and Spence 2008; Koppen and Spence 2007a 2007b 2007c; Sinnett et al. 2007), in some cases involving semantically meaningful stimuli (Koppen et al. 2008), the present study involved categorization of representations in incongruent contexts. Participants were therefore required to activate two conceptual representations of the stimuli in order to perform the task. Further, participants were required to briefly hold those conflicting representations in working memory until the presentation of the auditory target. Several questions remain unanswered regarding the interactions and interference between olfactory and visual processes. For example, rather than reflecting low-level asymmetric inhibition between sensory systems, our findings might stem from asymmetric interference between working memory representations. The observed categorization advantage of representations originating from olfactory input could result from a bias in the activation of the olfactory representations due to, for example, the focus on olfaction in the experimental context. A way of addressing this question would be to perform the same experimental paradigm, comparing visual input to input in another non-visual (e.g., auditory) sensory modality. If such a study would find a categorization advantage for visual input, we would have more robust evidence for concluding that our findings of dominance of olfactory representations stems from differences between the olfactory and other non-visual sensory systems.

Our findings clearly show that, contrary to widely held notions in neurocognitive perception research, olfactory representations can dominate visual representations under conditions of equal task-relevance. While the notion of visual dominance is well-established (Colavita 1974; Hartcher-O'Brien et al. 2010; Koppen et al. 2008; Koppen and Spence 2007a, 2007b, 2007c; Sinnett et al. 2007; Spence 2009; Spence et al. 2012), and olfaction was traditionally viewed as a "primitive" or underdeveloped sense (see Laska 2016; McGann 2017 for reviews), our findings, along with recent cross-cultural evidence (Majid et al. 2018), provide reasons to reconsider such generalized hierarchical views.

\section{Conclusions}

This study shows that, contrary to widely-held notions of visual dominance, olfactory processing can dominate visual processing under conditions of equal task-relevance and when differences in sensory encoding speed are controlled for. It extends earlier research by investigating cross-modal interference 
on the category level, using a categorization task with cross-modal interference. As such, it provides a novel paradigm for perceptual interference and competition for processing resources across sensory systems. Our findings provide support for the notion that visual behavioral categorization is especially disrupted by contextually incongruent odors. We speculate that incongruent odors might be particularly alerting, thereby facilitating further processing in other senses.

\section{Acknowledgments}

This work was supported by The Swedish Foundation for Humanities and Social Sciences (M140375:1) to M.L., and by the Knut and Alice Wallenberg Foundation (KAW 2016:0229) to J.K.O.

\section{References}

Alexander JE, Porjesz, B, Bauer LO, Kuperman S, Morzorati S, O’Connor SJ, ... Polich J. 1995. P300 hemispheric amplitude asymmetries from a visual oddball task. Psychophysiology. 32(5): 467-475.

Aristotle. 2010. On Sense And The Sensible. Whitefish (MT): Kessinger Publishing, LLC.

Colavita FB. 1974. Human sensory dominance. Perception \& Psychophysics. 16(2): 409-412.

Delorme A, Makeig S. 2004. EEGLAB: an open source toolbox for analysis of single-trial EEG dynamics including independent component analysis. Journal of Neuroscience Methods. 134(1): 921.

Dematte ML, Sanabria D, Spence C. 2008. Olfactory Discrimination: When Vision Matters? Chemical Senses. 34(2): 103-109.

Desimone R, Duncan J. 1995. Neural Mechanisms of Selective Visual Attention. Annual Review of Neuroscience, 18(1), 193-222.

Donchin E. 1981. Surprise!? Surprise? Psychophysiology. 18(5): 493-513.

Doty RL, Cameron EL. 2009. Sex differences and reproductive hormone influences on human odor perception. Physiology \& Behavior. 97(2): 213-228.

Duncan J. 1996. Cooperating brain systems in selective perception and action. In: Thosio I, McLelland $\mathrm{JL}$. editors. Attention and performance 16: Information integration in perception and communication. Cambridge (MA): The MIT Press. p. 549-578.

Falkenstein M, Hohnsbein, J, Hoormann, J. 1993. Late visual and auditory ERP components and choice reaction time. Biological Psychology. 35(3): 201-224.

Falkenstein M, Hohnsbein J, Hoormann J. 1994. Effects of choice complexity on different subcomponents of the late positive complex of the event-related potential. Electroencephalography and Clinical Neurophysiology. 92(2): 148-160.

Falkenstein M, Hohnsbein J, Hoormann J, Blanke L. 1991. Effects of crossmodal divided attention on late ERP components. II. Error processing in choice reaction tasks. Electroencephalography and Clinical Neurophysiology. 78(6): 447-455.

Forste, S, Spence C. 2018. "What Smell?" Temporarily Loading Visual Attention Induces a Prolonged Loss of Olfactory Awareness. Psychological Science. 29(10): 1642-1652.

Frömer R, Maier M, Abdel Rahman R. 2018. Group-Level EEG-Processing Pipeline for Flexible Single Trial-Based Analyses Including Linear Mixed Models. Frontiers in Neuroscience. 12.

García-Larrea L, Cézanne-Bert G. 1998. P3, positive slow wave and working memory load: a study on the functional correlates of slow wave activity. Electroencephalography and Clinical Neurophysiology. 108(3): 260-273.

Gelman A, Hill J. 2006. Data Analysis Using Regression and Multilevel/Hierarchical Models. New York (NY): Cambridge University Press.

Gevins A, Smith ME, Le J, Leong H, Bennett J, Martin N, ... Whitfield S. 1996. High resolution evoked potential imaging of the cortical dynamics of human working memory. Electroencephalography and Clinical Neurophysiology. 98(4): 327-348. 
Gottfried JA, Dolan RJ. 2003. The nose smells what the eye sees: crossmodal visual facilitation of human olfactory perception. Neuron. 39(2): 375-386.

Hartcher-O'Brien J, Levitan C, Spence C. 2010. Extending visual dominance over touch for input off the body. Brain Research. 1362: 48-55.

Herrick CJ. 1933. Morphogenesis of the brain. Journal of Morphology. 54(2): 233-258.

Hohnsbein J, Falkenstein M, Hoormann J, Blanke L. 1991. Effects of crossmodal divided attention on late ERP components. I. Simple and choice reaction tasks. Electroencephalography and Clinical Neurophysiology. 78(6): 438-446.

Honda M, Barrett G, Yoshimura N, Ikeda A, Nagamine T, Shibasaki, H. 1996. Event-related potentials during paired associate memory paradigm. Electroencephalography and Clinical Neurophysiology/Evoked Potentials Section. 100(5): 407-421.

Höchenberger R, Busch NA, Ohla K. 2015. Nonlinear response speedup in bimodal visual-olfactory object identification. Frontiers in Psychology. 6.

Hörberg T. forthcoming. Fishermans Friend: An R-based tool for statistical analysis and visualization of high-density EEG-data.

Hörberg T, Olofsson JK. 2018. Visual-olfactory dominance. Retrieved from osf.io/7qnwu/.

Johnson R, Donchin E. 1985. Second thoughts: multiple P300s elicited by a single stimulus. Psychophysiology. 22(2): 182-194.

Kok A, De Jong HL. 1980. Components of the event-related potential following degraded and undegraded visual stimuli. Biological Psychology. 11(2): 117-133.

Kok A. 2001. On the utility of P3 amplitude as a measure of processing capacity. Psychophysiology. 38(3): 557-577.

Koppen C, Alsius A, Spence, C. 2008. Semantic congruency and the Colavita visual dominance effect. Experimental Brain Research. 184(4): 533-546.

Koppen C, Spence C. 2007a. Seeing the light: exploring the Colavita visual dominance effect. Experimental Brain Research. 180(4): 737-754.

Koppen C, Spence C. 2007b. Audiovisual asynchrony modulates the Colavita visual dominance effect. Brain Research. 1186: 224-232.

Koppen C, Spence C. 2007c. Spatial coincidence modulates the Colavita visual dominance effect. Neuroscience Letters. 417(2): 107-111.

Kuehn M. 2006. Immanuel Kant: Anthropology from a Pragmatic Point of View. Louden RB, editor. Cambridge (UK): Cambridge University Press.

Köster EP, Møller P, Mojet J. 2014. A "Misfit" Theory of Spontaneous Conscious Odor Perception (MITSCOP): reflections on the role and function of odor memory in everyday life. Frontiers in Psychology. 5.

Kuang, S., Zhang, T. 2015. Smelling directions: Olfaction modulates ambiguous visual motion perception. Scientific Reports, 4(1).

Kutas M, McCarthy G, Donchin E. 1977. Augmenting mental chronometry: the P300 as a measure of stimulus evaluation time. Science. 197(4305): 792-795.

Laska M. 2015. Busting a myth: humans are not generally less sensitive to odors than non-human mammals. Oral presentation at the Association for Chemoreception Sciences (AChemS), 37th Annual Meeting, Bonita Springs, Florida, 22-25 April 2015. Chemical Senses. 40(7): 535-668.

Lundström JN, Gordon AR, Alden EC, Boesveldt S, Albrecht J. 2010. Methods for building an inexpensive computer-controlled olfactometer for temporally-precise experiments. International Journal of Psychophysiology. 78(2): 179-189.

Majid A, Roberts SG, Cilissen L, Emmorey K, Nicodemus B, O'Grady L, ... Levinson SC. 2018. Differential coding of perception in the world's languages. Proceedings of the National Academy of Sciences. 115(45): 11369-11376.

McGann JP. 2017. Poor human olfaction is a 19th-century myth. Science. 356(6338): eaam7263. 
McGurk H, MacDonald J. 1976. Hearing lips and seeing voices. Nature. 264(5588): 746-748.

Ngo MK, Cadieux ML, Sinnett S, Soto-Faraco S, Spence C. 2011. Reversing the Colavita visual dominance effect. Experimental Brain Research. 214(4): 607-618.

Olofsson JK, Bowman NE, Gottfried JA. 2013. High and low roads to odor valence? A choice response-time study. Journal of Experimental Psychology: Human Perception and Performance. 39(5): 1205-1211.

Olofsson JK, Bowman NE, Khatibi K, Gottfried JA. 2012. A Time-Based Account of the Perception of Odor Objects and Valences. Psychological Science. 23(10): 1224-1232.

Olofsson JK, Hurley RS, Bowman NE, Bao X, Mesulam MM, Gottfried JA. 2014. A Designated Odor-Language Integration System in the Human Brain. Journal of Neuroscience. 34(45): 1486414873.

Olofsson JK, Gottfried JA. 2015. The muted sense: neurocognitive limitations of olfactory language. Trends in Cognitive Sciences. 19(6): 314-321.

Olofsson JK, Syrjänen E, Ekström I, Larsson M, Wiens S. 2018. "Fast" versus "slow" word integration of visual and olfactory objects: EEG biomarkers of decision speed variability. Behavioral Neuroscience- 132(6): 587-594.

Pause BM, Sojka B, Krauel K, Ferstl R. 1996. The nature of the late positive complex within the olfactory event-related potential (OERP). Psychophysiology. 33(4): 376-384.

Picton TW. 1992. The P300 Wave of the Human Event-Related Potential. Journal of Clinical Neurophysiology. 9(4): 456-479.

Polich, J. 2007. Updating P300: An integrative theory of P3a and P3b. Clinical Neurophysiology, 118(10), 2128-2148.

Polich J. 2011. Neuropsychology of P300. In: Kappenman ES, Luck SJ. Editors. The Oxford Handbook of Event-Related Potential Components. Oxford (UK): Oxford University Press.

Pontifex MB, Miskovic V, Laszlo S. 2017. Evaluating the efficacy of fully automated approaches for the selection of eyeblink ICA components: Eyeblink component identification. Psychophysiology. 54(5): 780-791.

Posner MI, Nissen MJ, Klein RM. 1976. Visual dominance: An information-processing account of its origins and significance. Psychological Review. 83(2): 157-171.

R Core Development Team. 2018. R: A Language and Environment for Statistical Computing. Vienna (Austria): R Foundation for Statistical Computing. Retrieved from http://www.R-project.org/.

Ruchkin DS, Sutton S. 1983. Positive Slow Wave and P300: Association and Disassociation. Advances in Psychology. 10: 233-250.

Ruchkin DS, Sutton S, Kietzman ML, Silver K. 1980. Slow wave and P300 in signal detection. Electroencephalography and Clinical Neurophysiology. 50(1-2): 35-47.

Seigneuric A, Durand K, Jiang T, Baudouin JY, Schaal B. 2010. The Nose Tells it to the Eyes: Crossmodal Associations between Olfaction and Vision. Perception. 39(11): 1541-1554.

Sinnett S, Spence C, Soto-Faraco S. 2007. Visual dominance and attention: The Colavita effect revisited. Perception \& Psychophysics. 69(5): 673-686.

Spence C. 2009. Explaining the Colavita visual dominance effect. Progress in Brain Research. 176: $245-258$.

Spence C, Parise C, Chen YC. 2012. The Colavita Visual Dominance Effect. In: Murray MM, Wallace MT. editors. The Neural Bases of Multisensory Processes. Boca Raton (FL): CRC Press. p. 529556.

Stan Development Team. 2017. Stan Modeling Language: User's Guide and Reference Manual (Version 2.17.0). Retrieved from mc-stan.org.

Stan Development Team. 2018. RStan: the R interface to Stan (Version R package version 2.17.3). Retrieved from http://mc-stan.org/.

Sutton S, Braren M, Zubin J, John ER. 1965. Evoked-potential correlates of stimulus uncertainty. Science. 150(3700): 1187-1188. 
Thomas-Danguin T, Sinding C, Romagny S, El Mountassi, F, Atanasova B, Le Berre E, ... Coureaud G. 2014. The perception of odor objects in everyday life: a review on the processing of odor mixtures. Frontiers in Psychology. 5.

Traunmüller H, Öhrström N. 2007. Audiovisual perception of openness and lip rounding in front vowels. Journal of Phonetics. 35(2): 244-258.

Verleger R. 1997. On the utility of P3 latency as an index of mental chronometry. Psychophysiology. 34(2): 131-156.

Welch RB, Warren DH. 1980. Immediate perceptual response to intersensory discrepancy. Psychological Bulletin. 88(3): 638-667.

Zelano C, Montag J, Khan R, Sobel N. 2009. A Specialized Odor Memory Buffer in Primary Olfactory Cortex. PLOS ONE. 4(3): e4965.

Zhang M, Weisse, VD, Stilla R, Prather SC, Sathian K. 2004. Multisensory cortical processing of object shape and its relation to mental imagery. Cognitive, Affective \& Behavioral Neuroscience. 4(2): 251-259.

Zhou W, Jiang Y, He S, Chen D. 2010. Olfaction Modulates Visual Perception in Binocular Rivalry. Current Biology. 20(15): 1356-1358. 